A new systems approach to quantitative estimation of financial risks of investment projects was proposed: an integral risk of the project as a whole for all its parameters at once and the risks for each of its parameters separately. At the same time, the very concept of the project risk has been generalized: instead of the conventional risk of unprofitability, a new, more general concept of the risk of unacceptably low project profitability has been introduced. Two levels of the project profitability were considered: a level acceptable to the investor and a realistically achievable level. Corresponding values of design parameters and indices of financial efficiency of the project were found for these levels. Based on the found values, relative margins of investment acceptability and risks of unacceptably low profitability of the project were calculated. A procedure of comprehensive assessment of the risk of unacceptable low profitability of the project for cases of high certainty and partial uncertainty has been developed. Explicit formulas for quantitative risk assessment of unacceptably low profitability of the project have been derived, ranges of values of all risks under consideration have been determined and appropriate recommendations have been given. Explicit formulas for calculating the values of project risks and dynamic points of project acceptability are convenient and useful for software implementation (for example, within the Monte Carlo method). For the Monte Carlo method and the method of scenarios, another alternative approach to assessing the integral risk of unacceptably low project profitability was proposed by the authors based on the direct calculation of unacceptable scenario values of any criterion of the project financial efficiency. A new index of financial efficiency of the project has also been introduced: a discounted period of acceptable return (discounted payback period of the project is its special case)

Keywords: explicit formula, calculation, project risk, situation, high certainty, partial uncertainty

\title{
DEVELOPMENT OF A SYSTEMS APPROACH TO ASSESSMENT OF INVESTMENT PROJECT RISKS: RISKS OF UNACCEPTABLY LOW PROJECT PROFITABILITY
}

\author{
Alexander Vasiliev \\ Correspondingauthor \\ $\mathrm{PhD}$, Associate Professor
}

Department of Mathematical and Computer Modelling

Odessa I. I. Mechnikov National University

Dvoryanskaya str.,2, Odessa, Ukraine, 65029

E-mail: av5111955@gmail.com

Nataliia Vasilieva

$\mathrm{PhD}$, Associate Professor

Department of Higher Mathematics

Odessa State Academy of

Civil Engineering and Architecture

Didrihsona str.,4, Odessa, Ukraine, 65029

Natalia Tupko

$\mathrm{PhD}$, Associate Professor

Department of Applied Mathematics

National Aviation University

Huzara Lyubomyra str., 1, Kyiv, Ukraine, 03058

Received date 12.01.2022 Accepted date 15.02.2022 Published date 25.02.2022
How to Cite: Vasiliev, A., Vasilieva, N., Tupko,N.(2022). Development of a systems approach to assessment of investment project risks: risks of unacceptably low project profitability. Eastern-European Journal of Enterprise Technologies, 1 (4 (115)), 77-86. doi: https://doi.org/10.15587/1729-4061.2022.252997

\section{Introduction}

Investments always refer to the future, often very distant future which is always associated with uncertainty. Risk is a partial uncertainty when some information on the future situation is known with a probability of less than one. The following four main levels of uncertainty can be distinguished [1]:

1) clear enough future: a level of high certainty, a real possibility of forming one scenario of the future;

2) alternate futures: the level of partial uncertainty, description of the future in a form of several discrete scenarios;

3 ) continuous uncertainty: the range of the futures, continuous spectrum of possible scenarios;

4) true ambiguity: the level of high uncertainty, almost complete absence of information on future prospects.

In today's complex, unstable world, there are a large number of obvious and hidden factors affecting the course of economic processes. It is often impossible to trace the interaction of these factors. Therefore, one has to make economic decisions in conditions of incomplete information, i.e. in a situation of full or partial uncertainty. In this regard, scientific problematics related to the application of the theory of decision-making in conditions of uncertainty and risk in the economy are relevant. Within the framework of this problem, studies aimed at developing new approaches and methods for quantifying the financial risks of investment projects under conditions of uncertainty are of interest.

\section{Literature review and problem statement}

Studies of many researchers are devoted to quantitative analysis of financial risks of investment projects. The advantages and disadvantages of the main methods of project risk 
assessment are analyzed in detail in [1],however, there are no ways to eliminate these shortcomings. Study [2] shows how principles of financial project forecasting and risk management can be used both to improve NPV forecasts and increase resilience to project solution risks. Such methods of project risk assessment as risk-adjusted analysis, sensitivity analysis, decision tree method, and option pricing models are consideredin [3]. Article [4] discusses in detail the method of adjusting the discount rate by taking into account the risk. A way to correct the main drawback of the method (growth of the project risk by the time of the project completion) was offered. However, another shortcoming of the method (i.e., its one-factor nature) cannot be corrected since it is a consequence of the very essence of the method. Article [5] discusses methodological approaches to investing in the company and reducing the negative impact of risks. The algorithm of expressing investment risks through related risks and assessment of these risks was defined. However, the proposed risk indices are too deterministic. A theory of expected effect is presented in [6]. It is a new theory of assessing the investment project effectiveness in conditions of risk and uncertainty. It is focused on the consideration of various scenarios of project implementation. Only a situation of partial uncertainty is considered there. Study [7] presents fundamental concepts of risk analysis based on recent studies (concepts, theories, frameworks, approaches, principles, methods, models, risk assessment). The behavior of the breakeven point of an investment project depending on the discount rate is studied in [8] with the help of sensitivity analysis. Instead of the breakeven point, it would be more useful to study the point of acceptability when the project has not zero profitability but profitability acceptable for the investor. Project risks are assessed indirectly in [9] using analysis of the financial stability of the project and there are no explicit formulas for calculating the risk values. Only a case of partial uncertainty is considered. On the contrary, only the case of high certainty is considered in [10]. Project risks are also assessed indirectly, without the use of explicit formulas. An attempt is made in [11] to combine the analysis of breakeven production with the analysis of sensitivity and the method of scenarios. However, breakeven analysis is applied in the static version and the method of scenarios is also used in a simplified form. A mechanism of accounting for the project risk is studied in [12] using a new efficiency index, the average internal rate of return (AIRR). The objective consists of adjustment of preferences by indices of net present value (NPV) and AIRR. However, this will not be easy to achieve in the case of multiplicity of values of internal rate of return. An aggregate index of risk of the project money flows measuring the impact of risks on the flow of payments was developed in [13] using the method of expert evaluations. The disadvantage of such an index consists in the lack of objectivity as expert assessments are based on the subjective choice of specialists. A dynamic analysis of breakeven production was used in [14] to solve a practical money-saving problem. Sensitivity analysis was then used to establish the effect of parameters and the risks associated with them. The main disadvantages of this method consist of its one-factor nature and determinism. The method of risk-adjusted discount rate is used in [15] to estimate stochastic negative flows of project payments. The proposed approach is not universal: it is more suitable for estimating money flows with opposite signs. Article [16] is devoted to studying using the method of scenarios of a concrete high-risk project. The study has remained uncompleted. Apparently, a more powerful Monte Carlo method should have been used.
A model of the project efficiency index was constructed in [17] on the basis of the methods of hierarchy analysis, fuzzy set theory, and expert survey. The proposed approach is interesting but its objectivity is questionable since it is largely based on essentially subjective methods of expert evaluation. A new approach to rating the design parameters by their risks of unprofitableness in descending order was proposed in [18]. Risks are assessed indirectly by the values of corresponding margins of the investment breakeven. The proposed approach is compared with conventional analysis of sensitivity based on finding the elasticities of the project efficiency criterion by main project parameters. In addition to the above-mentioned shortcomings, all studies dedicated to the quantitative assessment of project risks have another common shortcoming. They assess the risk of project unprofitableness. At the same time, it is implicitly assumed that the breakeven level of the project's profitability, i.e. zero profitability, is acceptable. However, in reality, investors are unlikely to be interested in zero-income projects. Therefore, assessment of the risk of unacceptably low profitability of the project is relevant and promising from a practical point of view. In this case, investors set the acceptable level of project profitability in an absolute or relative form.

The study object consists of methods of quantitative analysis of own financial risk of investment projects for the first and second levels of uncertainty. This type of method is the most well-known among the methods used in practice: the method of discount rate adjustment, the method of reliable equivalents, the sensitivity analysis of project efficiency criteria, the scenario method, the decision tree method, the Monte Carlo method. However, all of these methods have significant drawbacks and limitations. Among existing conventional approaches, there is no universal method of analysis of the financial risk of the project suitable for any situation.

Therefore, it seems appropriate to conduct a study to elaborate a unified systems approach to assessing financial risks of investment projects suitable for both the case of high certainty and the case of partial uncertainty. Besides, such an approach should be applicable to the assessment of the integral risk of the project as a whole and the risks for main project parameters in particular.

\section{The aim and objectives of the study}

The study objective consisted in the development of a unified systems approach to quantitative assessment of the risk of unacceptably low profitability of investment project based on analysis of financial stability of the project in terms of its parameters and criteria of financial efficiency.

To achieve this objective, it was necessary to solve the following tasks:

- to derive explicit formulas for assessing the risks of unacceptably low profitability of the project in terms of its individual parameters in a situation of high certainty and find ranges of values of these risks;

- to derive explicit formulas for assessing integral risks of unacceptably low profitability of the project in a situation of high certainty and find the range of values of these risks;

- to derive explicit formulas for assessing the risks of unacceptably low profitability of the project in a situation of partial uncertainty and find the range of values of these risks;

- to derive explicit formulas for alternative assessment of integral risks of unacceptably low profitability of the project in a situation of partial uncertainty and find ranges of values of these risks. 


\section{The study materials and methods}

Principles of the decision theory in conditions of uncertainty and dynamic version of the breakeven analysis of project production [3, 8-10] have formed a methodological basis for the development of a new integrated approach to assessing the financial risk of projects. Assessment of the financial stability margin of the project is an integral part of the new approach. Breakeven margins have been reviewed by many authors. However, all studies on this issue were conducted almost exclusively as a part of conventional static breakeven analysis without taking into account the time value of money. Each investment project has a certain length of time. Therefore, when analyzing the project profitability, the concept of the time value of money can no longer be ignored. The concepts of financial stability margin of the project should be based on a dynamic breakeven analysis in which the index of profitability instead of profit is one of the discounted indices of project efficiency (NPV, PI, IRR). Concepts of investment breakeven margin and project acceptability for a dynamic case were introduced in $[9,10]$.

Before deriving explicit formulas for assessing the risk of unacceptably low profitability of the project, it was necessary to define the risk concept. The integral financial risk of the project's unprofitableness is interpreted in the conventional approach as a possibility of deviation of actual project results from breakeven results $[2,3,6,7]$. Integral risk of unacceptably low profitability of the project was interpreted in the proposed approach as a possibility of deviation of actual results of the project from those acceptable to the investor or project management. This interpretation of the integral financial risk of the project is suitable both in the case of high certainty and in the case of partial uncertainty. In the second case, the mathematical expectation of risk scenario values should be considered.

Quite similarly, the risk of unacceptably low profitability of the project for any of its parameters was interpreted as the possibility of deviation of the actual parameter value from the dynamic point of acceptability of the investment project (IP) for this parameter. In this case, the actual value of the parameter can be both worse and better than the acceptable value. In the case of partial uncertainty, it is necessary to move to mathematical expectations of scenario risks.

Conventional risk of the unprofitableness of an IP is a special case of the risk of unacceptably low profitability of the project if a breakeven level of profitability is considered the acceptable level.

\section{The results obtained in the studies of risks of} unacceptably low profitability of the investment project

5. 1. Assessment of the risk of unacceptably low profitability of the project by its main parameters in a situation of high certainty

First, we considered a somewhat idealized situation from a practical point of view when values of all project payments are known with probability close to one. The risk of unacceptably low profitability of the project was assessed by analyzing its financial stability in terms of individual project parameters or values of discounted indices of financial efficiency of the IP. Suppose that the net present value (NPV) index determined by the following formula [2,3] is chosen as an index of financial efficiency of the project:

$$
N P V=-I_{0}+\sum_{t=1}^{n} \frac{C F_{t}}{(1+i)^{t}},
$$

where $I_{0}$ is for initial investments in the IP; $t$ is for the number of the current period of the IP; $n$ is for the quantity of IP periods; $C F_{t}$ is for the amount of net income from the IP operation in the period $t ; i$ is for the discount rate of payments (cost of the IP capital).

Suppose that the following levels of profitability of the investment project required for analysis are set [10]:

1. The level of investment acceptability of the project. This level of absolute profitability can be set as follows:

$$
N P V \geq N P V_{*}>N P V_{0}>0,
$$

where $N P V$ is for an index of the value of the project calculated by (1); $N P V_{*}$ is for the lower limit of values of the absolute profitabilityof the IP acceptable to the investor; $N P V_{0}$ is for a relatively small positive number (e.g., $N P V_{0}=0.1 \cdot N P V_{*}$ ).

2 . Really (actually) achievable level of absolute profitability of IP in accordance with the expected flow of its payments:

$$
N P V=N P V_{p},
$$

where $N P V_{p}$ is for the value of the planned flow of IP payments found from (1).

Remark 1. If the lower acceptable limit $N P V_{*}=0$ is set for the 1st level of profitability, then the investment breakeven level of the project is obtained as a special case.

Let the flow of net incomes from the IP operation form a simple constant annuity postnumerando with payments having a structure of the form $[3,8,10]$ :

$$
C F_{t}=(Q(c-v)-F C-d e p)(1-\tau)+d e p=\text { const },
$$

where $Q$ is for the volume of production (sales) of homogeneous products for 1 IP period; $c$ is for price per product unit; $v$ is for specific variable production costs; $F C$ is for total fixed production costs for 1 IP period; dep is for depreciation deductions for 1 IP period; $\tau$ is for the income tax; $t$ is for the number of the current IP time period; $n$ is for the quantity of IP periods; $t=\overline{1, n}$.

Then, to calculate values of the NPV index of the project with a zero residual value of equipment, the formula from [3, 8, 10] can be applied:

$$
N P V=-I_{0}+\left(\begin{array}{l}
(Q(c-v)-F C-d e p) \times \\
\times(1-\tau)+d e p
\end{array}\right) a(n ; i),
$$

in which the discount factor for unit annuity for $n$ periods at a rate $i$ is defined as follows:

$$
a(n ; i)=\left(1-(1+i)^{-n}\right) / i .
$$

Values of design parameters corresponding to the lower limit of profitability acceptable for the project investor (level 1) were found. These values were determined from the equation:

$$
N P V=N P V_{*},
$$

where the value of $N P V_{*}$ is given in (2) and the left side of equation (7) has the form (5). 
Solution of equation (7) with respect to the $Q$ parameter with fixed values of remaining project parameters results in [10]:

$$
Q_{*}=\frac{1}{c-v}\left(\frac{1}{1-\tau}\left(\frac{I_{0}+N P V_{*}}{a(n ; i)}-d e p\right)+d e p+F C\right) .
$$

The volume $Q^{*}$ of production (sales) of IP products for 1 period is called the dynamic point of acceptability of the project in terms of parameter $Q$. If the level of acceptable profitability $N P V_{*}$ specified in (2) is increased, then volume $Q_{*}$ will also grow. Similarly, the dynamic points of acceptability of the project according to parameters $c, v$ and $F C$ can be found from equation (7), respectively [10]:

$$
\begin{aligned}
& c_{*}=v+\frac{1}{Q}\left(\frac{1}{1-\tau}\left(\frac{I_{0}+N P V_{*}}{a(n ; i)}-d e p\right)+d e p+F C\right), \\
& \nu_{*}=c-\frac{1}{Q}\left(\frac{1}{1-\tau}\left(\frac{I_{0}+N P V_{*}}{a(n ; i)}-d e p\right)+d e p+F C\right), \\
& F C_{*}=Q(c-v)-\frac{1}{1-\tau}\left(\frac{I_{0}+N P V_{*}}{a(n ; i)}-d e p\right)-d e p .
\end{aligned}
$$

As the value of $N P V_{*}$ grows, value $c *$ of the acceptable price also grows while the acceptable costs $v_{*}, F C *$ decrease.

Remark 2. Values of acceptability points can be obtained using (8)-(11) exactly, moreover, «manually». The same values can be found approximately as roots of equation (7) using any application package. With a one-time finding of acceptability points, the choice of the method for their calculation is of no fundamental importance. However, if it is required to find these points repeatedly in a computer program that implements the scenario method or the Monte Carlo method, then, in this case, it is much easier to program explicit formulas (8) to (11) than a resort to the software package each time.

Now it is possible to define concepts of investment acceptability margin of the project. The following values [10] can be called relative margins of investment acceptability of the project in terms of parameters $Q, c, v$, and $F C$, respectively:

$$
\begin{aligned}
& \beta_{Q}=\frac{Q_{p}-Q_{*}}{Q_{p}}=1-\frac{Q_{*}}{Q_{p}}, \\
& \beta_{c}=\frac{c_{p}-c_{*}}{c_{p}}=1-\frac{c_{*}}{c_{p}}, \\
& \beta_{v}=\frac{v_{*}-v_{p}}{v_{*}}=1-\frac{v_{p}}{v_{*}}, \\
& \beta_{F C}=\frac{F C_{*}-F C_{p}}{F C_{*}}=1-\frac{F C_{p}}{F C_{*}},
\end{aligned}
$$

where $Q_{p}, c_{p}, v_{p}, F C_{p}$ are real (actual) values of the IP parameters; $Q_{*}, c_{*}, v_{*}, F C_{*}$ are dynamic points of the project acceptability in terms of $Q, c, v, F C$ parameters respectively defined by formulas (8) to (11).

It is also possible to determine the dynamic point of acceptability and the margin of investment acceptability of the project by parameter $i$ (discount rate) but this can be done for an arbitrary flow of project payments.

The margin values (12) to (15) can be negative if actual values of some IP parameters turn out to be worse than the corresponding acceptance points. If the IP allows for the possibility of interactive intervention in the course of its implementation, then one can try to adjust the values of its parameters so that the values of all margins (12) to (15) become positive and as large as possible. If relative margins (12) to (15) are positive, then the IP has a certain margin of financial stability according to the listed parameters. Moreover, the greater the value of the relative margin for some project parameter, the less the risk of unacceptably low profitability of the project associated with this parameter.

Values of the risk of unacceptably low profitability of the IP for the listed design parameters were determined as follows:

- risk in terms of the production volume parameter for one IP period:

$$
\gamma_{Q}=1-\beta_{Q}=Q_{*} / Q_{p}
$$

- risk in terms of the price parameter per unit of IP product:

$$
\gamma_{c}=1-\beta_{c}=c_{*} / c_{p}
$$

- risk in terms of the parameter of specific variable production costs:

$$
\gamma_{\mathrm{v}}=1-\beta_{\mathrm{v}}=\mathrm{v}_{p} / \mathrm{v}_{*}
$$

- risk in terms of the parameter of total fixed production costs for one IP period:

$$
\gamma_{F C}=1-\beta_{F C}=F C_{p} / F C_{*}
$$

Ranges of values of introduced risks were found. At $Q_{p} \geq Q_{*}$, i.e., with production volumes acceptable for the investor for 1 IP period, $\gamma_{Q} \in(0 ; 1]$ was obtained and the risk reaches its maximum value of $Q_{p}=Q_{*}$ (while margin $\beta_{Q}=0$ ). Now, let $0<Q_{0} \leq Q_{p}<Q_{*}$, i.e., the real volume of production be less than acceptable but still does not cross the breakeven limit, where the breakeven value of $Q_{0}$ can be obtained by setting $N P V_{*}=0$ in (8). In this case, $\gamma_{Q} \in\left(1 ; Q_{*} / Q_{0}\right.$ ]. Thus, for any breakeven value of the parameter $Q_{p} \geq Q_{0}$ : $\gamma_{Q} \in\left(0 ; Q_{*} / Q_{0}\right]$. Risk (16) is maximum at $Q_{p}=Q_{0}$ and decreases with the growth of $Q_{p}$.

Similarly, for $c_{p} \geq c_{0}$, i.e. for breakeven price values, $\gamma_{c} \in\left(0 ; c_{*} / c_{0}\right]$, where the breakeven value $c_{0}$ can be obtained from (9) at $N P V_{*}=0$. Risk (17) decreases with price growth and is maximum at $c_{p}=c_{0}$. For price values acceptable to the investor, the maximum risk value (17) is equal to 1 when $\operatorname{margin} \beta_{\mathrm{c}}=0$.

Further, at $v_{p} \leq v_{0}$, i.e., for breakeven values of specific variable production costs $\gamma_{v} \in\left(0 ; v_{0} / v_{*}\right]$, where the breakeven value of $v_{0}$ can be obtained from (10) at $N P V_{*}=0$. Risk (18) decreases with a decrease in specific variable production costs and is maximum at $v_{p}=v_{0}$. For values of price acceptable to the investor, maximum risk value (18) is equal to 1 when margin $\beta_{v}=0$.

At $F C_{p} \leq F C_{0}$, i.e. for breakeven values of total fixed production costs for 1 IP period: $\gamma_{F C} \in\left(0 ; F C_{0} / F C_{*}\right]$, where breakeven value $F C_{0}$ can be obtained from formula (11) at $N P V_{*}=0$. Risk (19) decreases with a decrease in total fixed production costs and is maximum at $F C_{p}=F C_{0}$. For acceptable values of $F C_{p} \leq F C_{*}$, the maximum risk value is 1 when the margin $\beta_{F C}=0$.

If the value of any of the risks (16)-(19) exceeds its upper limit, this means that the project is unprofitable according to the corresponding parameter and should be rejected. 
Based on the risk values (16)-(19), it is possible to build a rating of IP parameters in descending order of the risk of unacceptably low profitability of the project. The parameters at the top of this ranking (especially those with poor predictive behavior) should be given increased attention due to their potential to interfere with the successful implementation of the project.

Remark 3. In the study of the risk of the project for each of its parameters, the case of production of only homogeneous IP products was considered. However, all concepts introduced in the paper can be used with appropriate adjustments for the case of multi-product manufacture. Only the proceeds from the sale of all types of IP products [9] must be considered instead of the production volume parameter.

\section{2. Assessment of integral risks of unacceptably low} profitability of the project as a whole in a situation of high certainty

If the flow of IP payments is arbitrary, i.e., it is not a simple constant annuity of the form (4), then it is also possible to introduce the concept of IP investment suitability margins. With their help, it is possible to assess the risk of unacceptably low profitability of the project as a whole in all its parameters at once. However, values of margins will be determined in this case by the values of integral indices of financial efficiency of the project and not by values of the design parameters.

Relative margins of investment eligibility of the IP were determined as follows:

- according to $N P V$ values:

$$
\beta_{N P V}=\frac{\left(N P V_{p}-N P V_{*}\right)}{N P V_{p}}=1-\frac{N P V_{*}}{N P V_{p}},
$$

where the $N P V_{*}$ value of the lower limit of the IP profitability acceptable to the investor is given in (2) and the $N P V_{p}$ value was found for the planned arbitrary flow of project payments according to (1);

- according to values of the PI (profitability index):

$$
\beta_{P I}=\frac{\left(P I_{p}-P I_{*}\right)}{P I_{p}}=1-\frac{P I_{*}}{P I_{p}},
$$

where $P I_{*}=1+N P V_{*} / I_{0} ; P I_{p}=1+N P V_{p} / I_{0}$, the $N P V_{*}$ value is given in (2) and the $N P V_{p}$ value is calculated according to (1). If the investor has initially set the level of IP profitability acceptable to him in a relative form, then the initial boundary value of acceptable relative IP profitability will be $P I_{*}$, and the value of $N P V *$ can be found using the formula: $N P V_{*}=\left(P I_{*}-1\right) \cdot I_{0}$;

- according to values of the index of internal rate of acceptable return (IRAR) introduced in [9]:

$$
\beta_{I R A R}=\frac{\left(I R A R_{p}-C C\right)}{I R A R_{p}}=1-\frac{C C}{I R A R_{p}},
$$

where $C C$ is for the cost of capital of an IP, $I R A R_{p}$ is the root of equation (7) for parameter $i$ (discount rate):

$$
N P V(I R A R) \equiv-I_{0}+\sum_{t=1}^{n} \frac{C F_{t}}{(1+I R A R)^{t}} \equiv N P V_{*}
$$

- according to values of the new index of a discounted period of acceptable return (DPAR) determined in this work:

$$
\begin{aligned}
& \beta_{D P A R}=\frac{\left(n-D P A R_{p}\right)}{n}=1-\frac{D P A R_{p}}{n}, \\
& D P A R_{p} \approx(m-1)+\frac{N P V_{*}+I_{0}-\sum_{t=1}^{m-1} C F_{t} /(1+i)^{t}}{C F_{m} /(1+i)^{m}},
\end{aligned}
$$

where $m$ is the number of the project period in which its absolute profitability reaches the $N P V *$ value specified in (2) for the first time. In the case of rental flow of IP payments when a more accurate formula can be applied:

$$
D P A R_{p}=-\ln \left(1-i \frac{\left(I_{0}+N P V_{*}\right)}{R}\right) / \ln (1+i) .
$$

With $N P V_{*}=0,(25),(26)$ are known formulas for the index of project efficiency in the discounted payback pe$\operatorname{riod}(\mathrm{DPP})$.

Values of risks of unacceptably low IP profitability according to the listed integral indices of financial efficiency were determined as follows:

- risk in terms of $N P V$ :

$$
\gamma_{N P V}=1-\beta_{N P V}=\frac{N P V_{*}}{N P V_{p}}
$$

where the margin $\beta_{\mathrm{NPV}}$ is defined in (20);

- risk in terms of $P I$ :

$$
\gamma_{P I}=1-\beta_{P I}=\frac{P I_{*}}{P I_{p}}
$$

where the margin $\beta_{\mathrm{PI}}$ is defined in (21);

- risk in terms of IRAR:

$$
\gamma_{I R A R}=1-\beta_{I R A R}=\frac{C C}{I R A R_{p}}
$$

where the margin $\beta_{\text {IRAR }}$ is defined in (22);

- risk in terms of DPAR index:

$$
\gamma_{D P A R}=1-\beta_{D P A R}=\frac{D P A R_{p}}{n},
$$

where the margin $\beta_{D P A R}$ is defined in (24).

Ranges of risk values were found (27)-(30).

Let $N P V_{p} \geq N P V_{*}$, i.e., absolute profitability of IP is not lower than an acceptable level. Then $\gamma_{N P V} \in(0 ; 1]$. Moreover, $\gamma_{N P V}=1$ at $N P V_{p}=N P V_{*}$, when the margin $\beta_{N P V}=0$. Now, let $0<N P V_{0} \leq N P V_{p}<N P V_{*}$, i.e., absolute profitability of IP is below an acceptable level but still positive. Then: $\gamma_{N P V} \in\left(1 ; N P V_{*} / N P V_{0}\right]$. At the same time, the margin is $\beta_{N P V}<0$. If the risk $\gamma_{N P V}>N P V_{*} / N P V_{0}$, then the IP is in a «dangerous» zone, close to the unprofitable level. The project is unprofitable at $\gamma_{N P V}<0$ and should be rejected. Thus, for any «safe» value $N P V_{p} \geq N P V_{0}: \gamma_{N P V} \in\left(0 ; N P V_{*} / N P V_{0}\right]$.

Risk (27) is maximum at $N P V_{p}=N P V_{0}$ and decreases with $N P V_{p}$ growth.

For any breakeven value of the index $P I_{p} \geq 1: \gamma_{P I} \in\left(0 ; P I_{*}\right]$.

Risk (28) is maximum at $P I_{p}=1$ and decreases with an increase in $P I_{p}$. When $\gamma_{P I}>P I_{*}$, the IP is unprofitable and should be rejected. If values of $P I_{p} \geq P I_{*}$ are acceptable for the investor, the maximum risk value is equal to 1 when the margin $\beta_{P I}=0$. 
For any breakeven IP, i.e. for $C C \leq I R R_{p}$, when $I R R_{p}$ is the real value of the internal rate of return of the IP: $\gamma_{I R A R} \in\left(0 ; I R R_{p} / I R A R_{p}\right]$.

The risk (29) is maximum at $I R R_{p}=C C$ (breakeven level of IP profitability) and decreases with the growth of $I R A R_{p}$. If $\gamma_{I R A R}>I R R_{p} / I R A R_{p}$, the project is unprofitable and should be rejected. With values of $I R A R_{p} \geq C C$ acceptable for the investor, the maximum risk value is equal to 1 , when the $\operatorname{margin} \beta_{I R A R}=0$.

If $D P A R_{p} \leq n$, i.e. when the IP profitability is not less than that acceptable to the investor, $\gamma_{D P A R} \in(0 ; 1]$. Moreover, the risk (30) is maximum at $D P A R_{p}=n$, when the margin $\beta_{D P A R}=0$. If $\gamma_{D P A R}>1$, then $N P V_{p}<N P V_{*}$, i.e., the IP profitability is below the level acceptable to the investor.

When comparing two alternative IPs, there is a concept of the Fisher point [3], i.e., such a discount rate $i_{\mathrm{F}}$ at which values of the NPV index of these projects coincide (the $N P V$ graphs of the projects intersect). At discount rates less than $i_{F}$, i.e., at $C C<i_{F}$, there is a contradiction between preferences in terms of NPV and IRAR. This contradiction remains valid for risks (27) and (29) as well. Therefore, it is advisable to introduce the concept of cumulative integral risk of unacceptably low profitability of the project for all four main criteria of IP's financial efficiency simultaneously. First, the risks (27) to (30) were normalized:

$$
\begin{aligned}
& \eta_{N P V}=\gamma_{N P V} /\left(\gamma_{N P V}^{2}+\gamma_{P I}^{2}+\gamma_{I R A R}^{2}+\gamma_{D P A R}^{2}\right)^{\frac{1}{2}}, \\
& \eta_{P I}=\gamma_{P I} /\left(\gamma_{N P V}^{2}+\gamma_{P I}^{2}+\gamma_{I R A R}^{2}+\gamma_{D P A R}^{2}\right)^{\frac{1}{2}}, \\
& \eta_{I R A R}=\gamma_{I R A R} /\left(\gamma_{N P V}^{2}+\gamma_{P I}^{2}+\gamma_{I R A R}^{2}+\gamma_{D P A R}^{2}\right)^{\frac{1}{2}}, \\
& \eta_{D P A R}=\gamma_{D P A R} /\left(\gamma_{N P V}^{2}+\gamma_{P I}^{2}+\gamma_{I R A R}^{2}+\gamma_{D P A R}^{2}\right)^{\frac{1}{2}}
\end{aligned}
$$

Obviously, after normalization, values of all risks (31) to $(34)$ belong to the interval $(0 ; 1)$ (provided that all unprofitable projects are excluded). The lower the values of (31) to (34), the lower the risk of unacceptably low profitability of the project in terms of the corresponding integral index of the project's financial efficiency.

Next, the total risk of IP for all four indices of financial efficiency was determined as follows:

$$
\eta=\eta_{N P V}+\eta_{P I}+\eta_{I R A R}+\eta_{D P A R}
$$

When risks of two alternative PIs are assessed, the one with lower total risk (35) is preferable. Index (35) gives a correct assessment of the total risk of IP only on a condition that absolute profitability of IP is not lower than the level acceptable to the investor, i.e.: $N P V p \geq N P V_{*}$. For the projects that are close to unprofitable, the first term in (35) will clearly prevail over the others.

5. 3. Assessment of the risk of unacceptably low profitability of the project in a situation of partial uncertainty

Let values of IP payments $\left\{C F_{t}^{k}\right\}_{t=1}^{n_{k}}$ under scenario $k$ of its development be known with a probability of less than one:

$$
0<p_{k}<1, k=\overline{1, m} ; \sum_{k=1}^{m} p_{k}=1
$$

where $m$ is for the number of possible IP development scenarios.
Then the formula for calculating the value of the $N P V$ index for scenario $k$ of IP development takes the form [10]:

$$
N P V_{k}=-I_{0}^{k}+\sum_{t=1}^{n_{k}} \frac{C F_{t}^{k}}{\left(1+i_{k}\right)^{t}}, k=\overline{1, m}
$$

In (37), the meaning of the design parameters for scenario $k$ of IP development is the same as in (1).

By analogy with (2), (3), IP profitability levels for scenario $k$ have the following forms:

1. The level of investment acceptability of the project for its $k$-th scenario:

$$
N P V_{k} \geq N P V_{*}>N P V_{0}>0, k=\overline{1, m},
$$

where values of the $N P V$ index for its $k$-th scenario were calculated according to (35) and values of $N P V_{*}, N P V_{0}$ that were set a priori have the same meaning as in inequality (2).

2. Actual level of the project profitability determined by the flow of its payments specified by the scenario condition:

$$
N P V=N P V_{k}, k=\overline{1, m},
$$

where the value of $N P V_{k}$ is found from formula (37).

Let the flow of net incomes from the operation of the project according to its $k$-th probable scenario have the form of a simple constant annuity:

$$
\begin{aligned}
& C F_{t}^{k}=\left(Q_{k}\left(c_{k}-v_{k}\right)-F C_{k}-d e p_{k}\right)\left(1-\tau_{k}\right)+d e p_{k}, \\
& k=\overline{1, m} ; t=\overline{1, n_{k}},
\end{aligned}
$$

where the IP parameters for its probable scenario numbered $k$ have the same meaning as in (4).

Then, to calculate the value of the NPV criterion for the $k$-th probable project scenario, a formula similar to (5) is applicable:

$$
\begin{aligned}
& N P V_{k}=-I_{0}^{k}+ \\
& +\left(\begin{array}{l}
\left(\begin{array}{l}
Q_{k}\left(c_{k}-v_{k}\right)- \\
-F C_{k}-d e p_{k}
\end{array}\right) \times \\
\times\left(1-\tau_{k}\right)+\operatorname{dep}_{k}
\end{array}\right) \cdot a\left(n_{k}, i_{k}\right), k=\overline{1, m,}
\end{aligned}
$$

where the unit annuity discount factor is as follows:

$$
a\left(n_{k}, i_{k}\right)=\frac{\left(1-\left(1+i_{k}\right)^{-n_{k}}\right)}{i_{k}} .
$$

For each possible IP scenario, it is possible to assess the risk of unacceptably low project profitability by one of its parameters proceeding from the value of the investment suitability margin for this parameter. First, it is necessary to find critical values of design parameters corresponding to the lower limit of the IP profitability acceptable to the investor from the equations:

$$
N P V_{k}=N P V_{*}, k=\overline{1, m},
$$

where the left parts of equations (43) take the form of (41).

When solving equations (43) with respect to the parameter of production volume with fixed values of the remaining parameters, the following can be obtained by analogy with (8): 


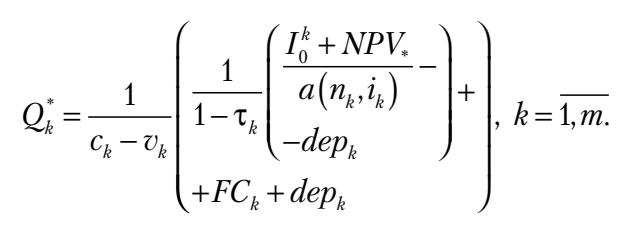

The project output volume (44) for one period is called a dynamic point of the project acceptability for this parameter for the $k$-th design scenario.

The following expression is called a relative margin of investment acceptability for one project period according to its probable $k$-th scenario in terms of the production volume parameter:

$$
\beta_{Q}^{k}=\frac{Q_{k}-Q_{k}^{*}}{Q_{k}}=1-\frac{Q_{k}^{*}}{Q_{k}}, k=\overline{1, m},
$$

where values of dynamic points of acceptability were taken from (44).

The risk of low IP profitability unacceptable to an investor for the $k$-th probable scenario in terms of parameter $Q$ was determined as follows:

$$
\gamma_{Q}^{k}=1-\beta_{Q}^{k}=\frac{Q_{k}^{*}}{Q_{k}}, k=\overline{1, m}
$$

Mathematical expectations of expressions (45), (46) for all possible IP scenarios take the form:

$$
\begin{aligned}
& M\left(\beta_{Q}\right)=\sum_{k=1}^{m}\left(1-Q_{k}^{*} / Q_{k}\right)^{*} p_{k}=1-M\left(\frac{Q^{*}}{Q}\right), \\
& M\left(\gamma_{Q}\right)=M\left(\frac{Q^{*}}{Q}\right)=1-M\left(\beta_{Q}\right) .
\end{aligned}
$$

The expression (48) characterizes the risk of unacceptably low profitability of the project as a whole in terms of parameter $Q$, i.e. for all possible scenarios.

Similarly, dynamic points of acceptability can be found and introduce the concepts of relative margins of investment acceptability and risks of unacceptably low profitability of the IP for its $k$-th probable scenario for other project parameters: $c, v, F C$.

Then their mathematical expectations can be found for all scenarios of the project and rank the project parameters in descending order of mathematical expectation of their risks. The parameters leading in such a rank should be given increased attention when implementing the project.

Risk (46) takes values from the following range:

$$
\gamma_{Q}^{k} \in\left(0 ; Q_{k}^{*} / Q_{k}^{0}\right], k=\overline{1, m},
$$

where $Q_{k}^{0}$ is the dynamic point of the project breakeven in terms of parameter $Q$ for its $k$-th probable scenario. Its value can be found using formula (44) at $N P V_{*}=0$.

When $\gamma_{Q}^{k}>Q_{k}^{*} / Q_{k}^{0}$, the IP is unprofitable for this scenario at number $k \in \overline{1, m}$. For breakeven values of the parameter, risk (46) is maximum at $Q_{k}=Q_{k}^{0}$ and decreases with an increase in the value of $Q_{k}$. For acceptable values of $Q_{k} \geq Q_{k}^{*}, k \in \overline{1, m}$, risk (46) takes values from $(0 ; 1)$ and is maximum at $Q_{k}=Q_{k}^{*}$, when margin (45) is equal to zero.

Risk (48) for all IP scenarios takes values from the interval $\left(0 ; M\left(Q^{*} / Q^{0}\right)\right)$, where $M\left(Q^{*} / Q^{0}\right)=\sum_{k=1}^{m} p_{k} \cdot Q_{k}^{*} / Q_{k}^{0}$. If
$M\left(\gamma_{Q}\right)>M\left(Q^{*} / Q^{0}\right)$, this means that the project is «on average» unprofitable in terms of the $Q$ parameter (that is, taking into account all scenarios) and should be rejected.

In the same way, it is possible to find ranges of change in risks of unacceptably low profitabilityof the IP for its $k$-th probable scenario for other project parameters, i.e. $c, v, F C$.

If amounts of project payments under the $k$-th probable scenario are arbitrary, i.e., the money flow is not a simple constant annuity of the form (40), then it is impossible to obtain project risk estimates in this case for each of its parameters separately. Parameter $i$ (the discount rate) is the exception. Only the integral risk of the project can be evaluated for all its parameters taken together using values of financial efficiency indices.

The integral risk of unacceptably low profitability of the project in terms of $N P V$ index for the $k$-th project scenario was determined as follows:

$$
\gamma_{N P V}^{k}=\frac{N P V_{*}}{N P V_{k}}, k=\overline{1, m},
$$

where the lower limit of the profitability acceptable to the investor in terms of $N P V$ indexis given in (38) and the value of the $N P V$ index for its $k$-th scenario was calculated using formula (41). If scenario value $N P V_{k} \geq N P V_{0}>0$, then $\gamma_{N P V}^{k} \in\left(0 ; N P V_{*} / N P V_{0}\right], k=1, m$. Risk (50) is maximum at $N P V_{k}=N P V_{0}$.

If the value of the risk (50) approaches the upper limit of the values range, this means that the IP under this scenario is close to being unprofitable. If the risk value is less than zero, then the project is completely unprofitable under this scenario.

Risk (50) is the less the greater the value of NPV for the $k$-th scenario of the project. The risk (50) values belong to the range $(0 ; 1)$ for acceptable $N P V_{k}$ values satisfying inequality (38).

To assess the integral risk of unacceptably low profitability of the project in terms of NPV index for all probable scenarios, mathematical expectation of risks (50) was found:

$$
\mu_{N P V}=\sum_{k=1}^{m} \gamma_{N P V}^{k} \cdot p_{k}
$$

where $0<p_{k}<1$ is for the probability of implementation of the $k$-th IP scenario, $k=\overline{1, m}$. $\ll$ Safe»values of risk (51) belong to the range $\left(0 ; N P V_{*} / N P V_{0}\right)$.

If the value of the risk (51) is close to the upper limit of the range, this means that the IP is «on average» close to being unprofitable. If the risk value is less than zero, then the project is «on average» unprofitable and should be rejected. The greater the value of the NPV index in the project scenarios the lower the risk. The risk (51) values belong to the interval $(0 ; 1)$ for acceptable $N P V_{k}$ values.

By analogy with (50), it is possible to estimate the integral risk of unacceptably low IP profitability for the $k$-th project scenario using other indices of IP's financial efficiency: PI, IRAR, DPAR. Then, by analogy with (51), we can proceed to mathematical expectations of corresponding scenario risks. Finally, the obtained mathematical expectations can be normalized as in (31) to (34) and the total IP risk can be defined as a sum of normalized integral risks for all four indices of the project efficiency taken together:

$$
\mu=\mu_{N P V}+\mu_{P I}+\mu_{I R A R}+\mu_{D P A R}
$$


After exclusion of all unprofitable IPs, values of each summand in (52) belong to the interval $(0 ; 1)$. When assessing the risk of two alternative IPs, the one with the lowest total risk (52) is preferable. Index (52) gives a correct assessment of the total IP risk only on the condition that the absolute IP profitability for all its scenarios is not lower than the level acceptable to the investor, i.e. $N P V_{k} \geq N P V_{*}$.

5. 4. Assessment of the project's integral risk in a situation of partial uncertainty by direct calculation of unacceptable scenario values

A fundamentally different approach to assessing the integral risk of unacceptably low IP profitabilitywas also considered for the case of partial uncertainty. This approach is based on a direct calculation of unacceptable scenario values of any index of the IP's financial efficiency. The expected value (EV) of the $N P V$ index for all IP scenarios is as follows:

$$
E V(N P V)=\sum_{k=1}^{m} N P V_{k} \cdot p_{k},
$$

where the value of the NPV index for its $k$-th scenario was found from (41); $0<p_{k}<1$ is for a probability of realization of the $k$-th IP scenario; $m$ is for the number of all IP scenarios. The greater the value (53), the greater the average absolute IP's profitability. If $E V(N P V)<0$, then IP is «on average» unprofitable and should be rejected. When $E V(N P V)>0$, analysis of the risk of unacceptably low IP profitability can be continued using the following new indices.

The expected unacceptability (EU) of the NPV index for all IP scenarios was determined as follows:

$$
E U(N P V)=\sum_{j=1}^{l}\left|N P V_{j}^{-}\right| \cdot p_{j},
$$

where $0<p_{j}<1 ; N P V_{j}^{-}<N P V_{*} ; j=\overline{1, l} ; l \leq m ; N P V_{*}$ is given in (38). The lower the value of (54) the lower risk of unacceptably low IP's profitability.

Next, variation of unacceptability (VU) of the NPV index was determined for all IP scenarios:

$$
V U(N P V)=\sum_{j=1}^{l}\left(E U(N P V)-N P V_{j}^{-}\right)^{2} \cdot p_{j} .
$$

The standard deviation of unacceptability (SDU) of the NPV index for all IP scenarios was set as follows:

$$
S D U(N P V)=\sqrt{V U(N P V)} .
$$

The coefficient of variation of unacceptability (CVU) of the NPV index for all IP scenarios was determined as follows:

$$
C V U(N P V)=S D U(N P V) / E U(N P V) .
$$

The lower the values of (54) to (57), the lower the risk of unacceptably low IP's profitability. The probability of implementing the IP scenario with unacceptably low profitability for the Monte Carlo method is as follows:

$$
P\left(N P V_{k}<N P V_{*}\right)=l / m ; l \leq m,
$$

and for the method of scenarios, it is equal to the sum of probabilities of scenarios for which the IP's profitability is below the acceptable value.
Finally, the expected unacceptability ratio (EUR) of the NPV index for all IP scenarios has the form:

$$
E U R(N P V)=\frac{E U(N P V)}{E U(N P V)+E A(N P V)},
$$

where $E A(N P V)=\sum_{j=1}^{r} N P V_{j}^{+} \cdot p_{j}$ is the expected acceptability $(\underline{E A})$ of the NPVindex for all IP scenarios, $N P V_{j}^{+}>N P V_{*}$; $j=\overline{1, r} ; r=m-l$. Values of the coefficient (59) belong to $[0 ; 1]$. The risk is maximum at $E A(N P V)=0$ (absence of acceptable values) and minimum at $E U(N P V)=0$ (absence of unacceptable values).

All risk indices (53) to (59) can also be determined for other integral indices of IP's financial efficiency: PI, IRAR, DPAR. These indices can be used to assess the risk of unacceptably low IP's profitability both in the method of scenarios and in the Monte Carlo method.

\section{Discussion of the results obtained in the study of risks of unacceptably low profitability of investment projects}

The proposed approach to assessing financial risks of IP has the following advantages compared to the conventional methods: complex nature, a more general concept of risk, availability of explicit formulas for calculating the risk values, knowledge of the ranges of risk value changes.

To demonstrate the distinctions and advantages of the proposed approach, an example of a concrete IP from [8] was considered. Let IP with payment flow (4) have the following basic values of parameters: $I_{0}=\$ 150,000 ; n=10$; $c_{p}=\$ 3.70 ;$ dep $=\$ 15,000 ; v_{p}=\$ 3.00 ; \quad F C_{p}=\$ 30,000 ; \quad i=0.1 ;$ $Q_{p}=100,000$ un.; $\tau=0.35$. A discrete version of sensitivity analysis was used in [8] to assess the influence of parameters on the IP results. Values of design parameters varied according to the rule: $\pm 20 \%$; $\pm 10 \%$ of the basic values. Next, magnitudes of the $N P V$ index changes were determined for the varied values of parameters. The greater the $N P V$ change the more influential the parameter and the greater the risk associated with this parameter. As a result, the following rank of parameters was obtained in descending order of their influence on the IP results and corresponding risks:

1) $c$ (price per product unit);

2) $v$ (specific variable costs);

3) $Q$ (production volume);

4) $F C$ (total fixed costs).

Now, let us explore the risks of unacceptably low profitability of this IP in terms of the main design parameters using the author's approach implemented as a program in Python language. Let the level of IP profitability acceptable to an investor be equal to $N P V_{*}=\$ 30.000$. Let us find dynamic points of IP acceptability for parameters $Q, c, v, F C$ according to (8)-(11): $Q_{*}^{*}=95.716$ un.; $c *=\$ 3.67 ; v *=\$ 3.03$; $F C_{*}=\$ 33.019$. For comparison, the dynamic breakeven point of this IP is $Q_{0}=84.986$ in terms of the $Q$ parameter.

Let us estimate the risks of unacceptably low profitability of this IP according to (16) to (19):
1) $\gamma_{c}=0.992$
2) $\gamma_{v}=0.990$;
3) $\gamma_{Q}=0.957$
4) $\gamma_{F C}=0.909$.

As can be seen, the ranks of IP parameters are the same for both approaches. However, due to the availability of explicit 
formulas, values of the risks themselves are also obtained and not the values indirectly related to them. It can be concluded from analysis of the risk values that values of all main IP parameters are critically close to their acceptability limits but still do not go beyond them. With conventional sensitivity analysis, such conclusions cannot be drawn since there are no explicit values of the risks themselves. An example of using another conventional version of sensitivity analysis based on finding elasticity of the criterion of financial efficiency with respect to design parameters was also considered [18]. Let basic values of the IP parameters with the flow of payments (4) be as follows: $I_{0}=2,000 ; n=6 ; c_{p}=50 ; d e p=100 ; v_{p}=40$; $F C_{p}=500 ; i=0.12 ; Q_{p}=200 ; \tau=0.24$.

Elasticities of the NPV indicesfor main IP parameters were found in [18] and the following rank of parameters was constructed in descending order of elasticities and the risks associated with them (elasticity values are given in brackets):

1) $c\left(E_{c}(N P V)=11.21\right)$

2) $v\left(E_{v}(N P V)=8.97\right)$

3) $Q\left(E_{Q}(N P V)=2.24\right)$;

4) $F C\left(E_{F C}(N P V)=0.56\right)$

Apart from this comparative indirect analysis of the influence of IP parameters, no more information on the project risks can be obtained. Using the proposed approach, let us study the risks of unacceptably low profitability of this IP for the level of the project profitability $N P V_{*}=2500$ acceptable to the investor. Let us find dynamic IP acceptability points for parameters $Q, c, v, F C$ according to (8)-(11): $Q_{*}^{*}=191$; $c_{*}=49.55 ; v_{*}=40.46 ; F C *=591.08$. For comparison, dynamic breakeven points of this IP are as follows: $Q_{0}=111 ; c_{0}=45.54$; $v_{0}=44.46 ; F C_{0}=1391.37$. Let us find values of the risk of unacceptably low profitability of this IP using (16) to (19):

1) $\gamma_{c}=0.991$

2) $\gamma_{v}=0.989$

3) $\gamma_{Q}=0.955$;

4) $\gamma_{F C}=0.846$.

Ratings of the IP parameter for both approaches are the same. It can be concluded from the analysis of the risk values that the values of all main IP parameters are critically close to their acceptability limits. Let us find the risks of the unprofitableness of this IP at $N P V *=0$ :

1) $\gamma_{c}=0.911$

2) $\gamma_{v}=0.9$

3) $\gamma_{Q}=0.555$

4) $\gamma_{F C}=0.359$

The risks of unprofitableness in all parameters are less (especially in terms of $Q$ and $F C$ ). With an increase in the level of IP profitability acceptable to the investor for $N P V_{*}=3,000$, all risks of unacceptably low profitability of this IP will become more than 1 . This means that the basic values of all main parameters of this IP will go beyond their acceptability limits.

Now, let us consider the state of affairs with an assessment of the integral risk of IP in the case of high certainty. Of all conventional methods, the discount rate correction method is mainly used for this purpose $[4,15]$. At the same time, the risk of IP as a whole is assessed either by in-house procedures or by an expert method, i.e. both are subjective methods. Moreover, the risk assessment is indirect. It finds its expression in the value of the premium (risk premium) to the basic discount rate. Thus, the main disadvantages of the method consist inits subjectivity and one-factor nature. There was another one: an incorrect growth of risk of the IP by the time of its completion (this drawback seems to be corrected [4]).
According to (27)-(30), it is possible to obtain an explicit numerical value of the integral IP risk with the help of the proposed approach. For example, for the latter IP, the integral risk of unacceptably low profitability in terms of NPV indexis as follows: $\gamma_{N P V}=N P V_{*} / N P V_{p}=2500 / 2786 \approx 0.897$, i.e., rather high.

For the case of partial uncertainty, there are effective methods of quantifying the project risks:the method of scenarios and the Monte Carlo method. However, firstly, they could not assess the IP risks by individual parameters, and, secondly, they assessed the risk of the project's unprofitableness. In the proposed approach, these methods were modified to assess the risks of unacceptably low profitability of IP. Now the scenario method can be used to measure risks in terms of parameters (48) and integral risk (51). In addition, the authors proposed another alternative approach (53) to (59) for assessing the integral risk of unacceptably low IP profitability. Let the parameters take the following values for an IP with scenario payment flows (40): $Q_{k} \in[150 ; 300]$; $c_{k} \in[35 ; 55] ; v_{k} \in[25 ; 40] ; F C_{k}=500 ; \operatorname{dep}_{k}=100 ; i_{k}=0.1 ; n_{k}=5$; $I_{0}^{k}=2000 ; \tau_{k}=0.18 ; m=1000 ; k=1,2, \ldots, m$. To assess the risks of this IP according to (53)-(59), the Monte Carlo method implemented as a program in the Python language was used. Two levels of IP profitability acceptable to an investor were considered. With $N P V_{*}=0$, the following values of indices (58), (59) of the integral risk of IP unprofitableness were obtained: $P\left(N P V_{k}<0\right)=0.24 ; E U R(N P V)=0.11$. These estimates can be obtained using the conventional approach. Since the values of the indices are relatively small, the investor is likely to agree to invest in this IP. At the level of acceptable profitability, $N P V_{*}=2000: P\left(N P V_{k}<2000\right)=0.48$; $\operatorname{EUR}(N P V)=0.18$. The situation has changed significantly. A risk-averse investor will not choose this IP for investment.

The considered examples show that the proposed approach, in comparison with conventional methods, provides more useful information on the IP risks for potential investors. Instead of the risk of IP unprofitableness, it is advisable to assess the risk of unacceptably low profitability of the project since it is significantly higher. This may affect the investor's decision about the project. This approach is in the investor's interests.

The risk assessment for each project parameter is inapplicable when there is an arbitrary flow of project payments. One can try to replace the arbitrary payment flow with an equivalent annuity flow of a special structure with averaged values of parameters. This is seen as a further study development line.

\section{Conclusions}

1. Explicit formulas have been obtained for assessing risks of unacceptably low profitability of the project for each of its parameters in a situation of high certainty. These formulas give a quantitative measure of the risks themselves rather than auxiliary values indirectly related to the IP risks. Ranges of risk values were found. The risks change in the interval of $(0 ; 1]$ for acceptable parameter values. The risks are greater than 1 for unacceptable values.

2. Explicit formulas have been derived for assessing integral risks of unacceptably low profitabilities for all main indices of the IP's financial efficiency in a situation of high certainty. The obtained estimates are more objective than the conventional ones. Ranges of risk values were found. For low-income projects, values of integral risks are greater 
than one. In order to coordinate the setting of the level of acceptable profitability of the project, a new index of the IP's financial efficiency was introduced. This index is a discounted period of acceptable return (DPAR). The DPAR index is more general than the well-known discounted payback period (DPP) index and coincides with it when the profitability level is set to zero. An explicit formula has been derived for assessing the total integral risk of IP in a situation of high certainty. This formula is useful when there are conflicting recommendations in terms of NPV and IRAR (presence of the Fisher point). However, it gives a correct assessment of total IP risk only on a condition that absolute IP's profitability is not lower than the level acceptable to the investor.

3. An explicit formula has been derived for assessing the IP risk in terms of the production volume parameter in a situation of partial uncertainty. Formulas for other design parameters can be obtained similarly. Such estimates are absent in the known conventional methods. An explicit formula has been derived for assessing the integral risk of unacceptably low profitability of IP in terms of NPV in a situation of partial uncertainty. A range of risk values was found: interval $(0 ; 1)$ for acceptable values of $N P V_{k}$ and $(1 ;+\infty)$ for unacceptable values. Formulas for other IP efficiency indices can be derived in a similar way. An explicit formula was also derived for assessing the total integral IP risk in a situation of partial uncertainty. It is correct for acceptable IP profitability values.

4. Explicit formulas have been derived for an alternative assessment of integral IP risks in terms of NPV in a situation of partial uncertainty. Unlike conventional formulas of this type, these formulas evaluate the integral risk of unacceptably low profitability of IP and not the risk of unprofitableness, i.e., they are more general. The range of values of risk indices (58), (59) is the interval $[0 ; 1]$. Similar formulas for other indices of financial efficiency of IP can be obtained in a similar way.

\section{References}

1. Keršytė, A. (2012). Investment risk analysis: theoretical aspects. Economics and management, 17 (3). doi:https://doi.org/10.5755/ j01.em.17.3.2099

2. Hopkinson, M. (2017). Net Present Value and Risk Modelling for Projects. Routledge, 180. doi: https://doi.org/10.4324/9781315248172

3. Gotze, U., Northcott, D., Schuster, P. (2015). Investment Appraisal. Methods and Models. Springer, 363. doi: https://doi.org/ 10.1007/978-3-662-45851-8

4. Carmichael, D. G. (2016). Adjustments within discount rates to cater for uncertainty - Guidelines. The Engineering Economist, 62 (4), 322-335. doi: https://doi.org/10.1080/0013791x.2016.1245376

5. Zholonko, T., Grebinchuk, O., Bielikova, M., Kulynych, Y., Oviechkina, O. (2021). Methodological Tools for Investment Risk Assessment for the Companies of Real Economy Sector. Journal of Risk and Financial Management, 14 (2), 78. doi: https://doi.org/ $10.3390 / \mathrm{jrfm} 14020078$

6. Smolyak, S. A. (2012). Investment Project Evaluation under Risk and Uncertainty Investment Project Evaluation under Risk and Uncertainty (Expected Effect Theory). Moscow, 158. doi:https://doi.org/10.2139/ssrn.2097349

7. Aven, T. (2015). Risk Analysis. John Wiley \& Sons, Ltd, 197. doi: https://doi.org/10.1002/9781119057819

8. Tarzia, D. A. (2016). Properties of the Financial Break-Even Point in a Simple Investment Project As a Function of the Discount Rate. Journal of Economic \& Financial Studies, 4 (02), 31-45. doi: https://doi.org/10.18533/jefs.v4i02.226

9. Vasil'ev, A., Vasil'eva, N., Tupko, N. (2017). Development of combined method for analysis of financial risks of investment project. Technology Audit and Production Reserves, 4 (4 (36)), 43-49. doi: https://doi.org/10.15587/2312-8372.2017.108527

10. Vasyliev, O., Vasylieva, N., Tupko, N. (2021). Reserves of investment acceptability of the project based on values of its parameters and efficiency criteria. Scientific Bulletin of Kherson State University. Series Economic Sciences, 43, 81-85. doi: https://doi.org/ 10.32999/ksu2307-8030/2021-43-12

11. Firescu, V., Branza, D. (2016). Cost volume profit, a managerial accounting technique. Scientific Bulletin - Economic Sciences, 15 (3), 25-34. URL: http://economic.upit.ro/repec/pdf/2016_3_3.pdf

12. Hazen, G., Magni, C. A. (2021). Average internal rate of return for risky projects. The Engineering Economist, 66 (2), $90-120$. doi:https://doi.org/10.1080/0013791x.2021.1894284

13. Mahmoud, H., Ahmed, V., Beheiry, S. (2021). Construction Cash Flow Risk Index. Journal of Risk and Financial Management, 14 (6), 269. doi: https://doi.org/10.3390/jrfm14060269

14. Nykamp, S., Bakker, V., Molderink, A., Hurink, J. L., Smit, G. J. M. (2013). Break-even analysis for the storage of PV in power distribution grids. International Journal of Energy Research, 38 (9), 1112-1128. doi: https://doi.org/10.1002/er.3106

15. Blaset Kastro, A. N., Kulakov, N. Y. (2020). Risk-adjusted discount rates and the present value of risky nonconventional projects. The Engineering Economist, 66 (1), 71-88. doi:https://doi.org/10.1080/0013791x.2020.1815918

16. Brzakovic, T., Brzakovic, A., Petrovic, J. (2016). Application of scenario analysis in the investment projects evaluation. Ekonomika Poljoprivrede, 63 (2), 501-513. doi: https://doi.org/10.5937/ekopolj1602501b

17. Prokopenko, T., Lavdanska, O., Povolotskyi, Y., Obodovskyi, B., Tarasenko, Y. (2021). Devising an integrated method for evaluating the efficiency of scrum-based projects in the field of information technology. Eastern-European Journal of Enterprise Technologies, 5 (3 (113)), 46-53. doi:https://doi.org/10.15587/1729-4061.2021.242744

18. Vasiliev, O., Vasilieva, N., \& Tupko, N. (2019). A new approach to formation of rating of parameters of investment project in terms of their risks. Scientific Bulletin of Kherson State University. Series Economic Sciences, 34, 153-156. doi: https://doi.org/10.32999/ ksu2307-8030/2019-34-33 